\title{
Überaktive Blase: Patienten profitieren von der Therapie mit Fesoterodin
}

_ In der FACT-Studie, in der erstmals bei Patienten mit überaktiver Blase zwei Antimuskarinika auf Überlegenheit miteinander verglichen wurden, schnitt Fesoterodin $\left(\right.$ Toviaz $^{\circledR}$ ) besser ab als Tolterodin [Herschorn S et al. BJU Int. 2010; 105: 5866]. Die Studienteilnehmer hatten über zwölf Wochen randomisiert, doppelblind $4 \mathrm{mg}$ Tolterodin retard $(n=684)$, Placebo $(n=334)$ oder Fesoterodin - zunächst $4 \mathrm{mg}$, nach einer Woche dann $8 \mathrm{mg}(\mathrm{n}=679)$ - erhalten.

Fesoterodin reduzierte die Zahl der Dranginkontinenz-Ereignisse pro Tag signifikant stärker als Tolterodin (-1,72 vs. -1,61, p<0,05). Auch die Änderung des Miktionsvolumens war signifikant größer (+32,9 ml vs. $+23,5$ ml, p<0,05). „Dass der Unterschied hier so deutlich ist, überrascht“, betonte Dr. Daniela Marschall-Kehrel, Frankfurt/Main.

In einer Post-hoc-Analyse zeigte sich basierend auf Patientenangaben zudem eine signifikante Überlegenheit von Fesoterodin gegenüber Tolterodin bei der durchschnittlichen Dranghäufigkeit, der

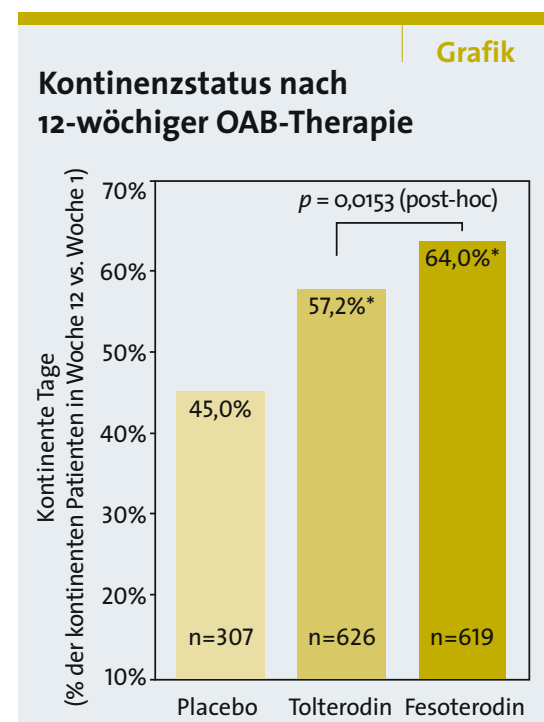

Die statistische Interferenz zwischen den Gruppen basierte auf dem CMH-Test stratifiziert nach Ausgangs-UUI-Quartilen. ${ }^{*} p<0,001$ vs. Placebo

Wahrnehmung des Blasenzustandes, dem Schweregrad des imperativen Harndrangs und der gesundheitsbezogenen Lebensqualität/Symptomlast (je $p<0,05$ ).

Darüber hinaus konnte bei signifikant mehr Patienten die Zahl der DranginkontinenzEreignisse von mindestens 1 zu Studienbeginn auf o in Woche 12 reduziert werden $(64,0$ vs. $57,2 \%, p<0,05)$. Zu den häufigsten Nebenwirkungen gehörten jeweils Mundtrockenheit (27,8\% bzw. $16,4 \%)$, Kopfschmerzen (5,6\% bzw. 3,4\%) und Obstipation (5,4\% bzw. 4,1\%). Sie waren jedoch mild und führten nur sehr selten zum Therapieabbruch.

Wie Marschall-Kehrel hervorhob, wird Fesoterodin kaum ins Gehirn aufgenommen und nicht über Cytochrom P450 metabolisiert, was vor allem für ältere und multimorbide Patienten sowie Kinder wichtig ist.

\section{pe}

Pressekonferenz „Erste Head-to-headStudie zum direkten Vergleich der beiden Antimuskarinika Fesoterodin (Toviaz ${ }^{\circledR}$ ) und Tolterodin (Detrusitol $\left.{ }^{\circledR}\right)^{\prime \prime}$, Berlin, 10. Februar 2010; Veranstalter: Pfizer Pharma

\section{Therapie der Erektilen Dysfunktion}

\section{Bedürfnisse beider Partner berücksichtigen}

Für eine optimale Behandlung der erektilen Dysfunktion (ED) sind nicht nur die Anliegen der Männer entscheidend, sondern auch die Perspektive der Partnerinnen. Die in Frankfurt am Main niedergelassene Urologin Dr. Daniela MarschallKehrel nimmt Stellung.

\section{? Inwiefern wird die Partnerschaft durch eine ED beeinflusst?}

Marschall-Kehrel: Zu einer Partnerschaft gehört meist auch der Wunsch nach einer

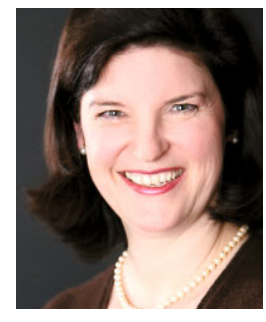

"Man sollte auch die Lebenssituation des Patienten berücksichtigen", sagt Dr. Daniela MarschallKehrel, Frankfurt. erfüllten Sexualität. Leidet der Mann an Erektiler Dysfunktion, tritt dieser wertvolle Aspekt der Paarbeziehung häufig in den Hintergrund. Zieht sich der Partner emotional und körperlich zurück, wird dies von der Frau häufig falsch interpretiert: Reicht ihre Attraktivität und Anziehungskraft für den Partner nicht mehr aus, um genügend Leidenschaft im Bett zu entwickeln? Sind seine Gefühle für sie nicht mehr intensiv genug?

\section{? Unterscheiden sich die Wïnsche von Frau- en und Männern an eine ED-Therapie? Marschall-Kehrel: Die meisten Männer mit nachlassender erektiler Funktion wün- schen sich eine Situation, die der vor Auf- treten der ED ähnelt. Sie möchten sich wieder „normal“ fühlen und möglichst we- nig an ihre Erkrankung erinnert werden. Die meisten Frauen wünschen sich zudem,}

dass sexuelle Aktivitäten unter der Therapie möglichst selbstbestimmt gestaltet werden können und wenig gefühlter Zeitdruck aufkommt.

\section{? Was empfehlen Sie diesen Paaren?} Marschall-Kehrel: Die Erfahrung zeigt, dass dem Wunsch nach zeitlicher Flexibilität zum Beispiel der länger wirksame PDE-5Hemmer Tadalafil entgegen kommen kann. Sexualität kann damit weitgehend gedanklich entkoppelt vom Zeitpunkt der Medikamenteinnahme stattfinden. Man sollte aber auch die Lebenssituation des Patienten berücksichtigen: Führt das Paar eine Wochenendbeziehung oder hat es nicht so häufig Geschlechtsverkehr, würde ich beispielsweise die Bedarfstherapie mit einem Wirkzeitfenster von bis zu 36 Stunden empfehlen. Paaren, denen es sehr wichtig ist, sich öfter als einmal in der Woche zu lieben, lege ich die einmal tägliche Therapie mit Tadalafil in niedriger Dosis ans Herz.

Nach Information von Lilly 